\title{
HETERODYNE INTERFEROMETRY IN THE INFRARED
}

\author{
C.H. TOWNES, M. BESTER, W.C. DANCHI, C.G. DEGIACOMI* , and L.J. \\ GREENHILL** \\ Space Sciences Laboratory and Physics Department \\ University of California at Berkeley \\ Berkeley, CA 94720 USA
}

\begin{abstract}
The University of California Infrared Spatial Interferometer (ISI) for the $10 \mu \mathrm{m}$ wavelength region is briefly described along with results obtained on prominent stars and on atmospheric phenomena. The system has two movable telescopes of $1.65 \mathrm{~m}$ aperture. It operates in principle like a modern radio interferometer, using heterodyne detection, $\mathrm{CO}_{2}$ laser local oscillators, RF delay lines, and lobe rotation to maintain a fixed-frequency fringe rate.

Rather extensive measurements have been made on atmospheric pathlength or phase fluctuation characteristics which show substantial deviations from the Kolmogorov-Taylor model, fortunat ely in a direction favoring adaptive optics, long baselines, and the use of infrared wavelengths. Outer scales as small as about 10 meters occur under good seeing conditions. Visibility results on 13 stars show that 6 of them have dust shells rather far from the star and give evidence for episodic emission of gas. Others of the 13 stars also vary with time, but are characterized by more continuous emission and dust formation near the stars at temperatures as high as $1300 \mathrm{~K}$.
\end{abstract}

\section{Introduction}

The University of California Infrared Spatial Interferometer (ISI) operates in the 10 $\mu \mathrm{m}$ wavelength region. It utilizes two Pfund-type telescopes of 1.65 meters aperture, each mounted in a movable trailer so that baselines can be varied, with the present installation, between 4 and 35 meters. Figure 1 is a schematic of one such trailer. Heterodyne detection of double sideband width of about $4 \mathrm{GHz}$ is utilized which allows an IF coaxial delay line to be used to obtain white light fringes. Two separate laser local oscillators are synchronized in phase and changed in relative frequency so that lobe rotation is obtained and fringes provided at some chosen audio frequency, typically 10 or $100 \mathrm{~Hz}$. Recent technical developments are described in the paper by Bester et al. in these proceedings.

\section{Atmospheric measurements}

Fluctuations in atmospheric pathlengths have a strong effect on the practicality of spatial interferometry in the optical or infrared regions from the earth's surface. The ISI telescopes are operating on Mount Wilson which usually has favorable seeing conditions, but nevertheless gives wide variations in effective sensitivity. Results are illustrated by Figure 2, where the fringe frequency is as narrow as one over the 2621 seconds of observation $(0.00038 \mathrm{~Hz})$ under very good seeing conditions and by Figure 3, where an observation of 262 seconds shows a fringe width (FWHM) of about $12 \mathrm{~Hz}$ due to atmospheric pathlength fluctuations under poor seeing conditions. If the seeing had been as good in the case of Figure 3 as it was for the case shown in Figure 2, the central peak would have been larger by a factor of 3000 , or

* Present address: Spectrospin, Zurich, Switzerland

** Present address: Harvard Smithsonian Center for Astrophysics, Cambridge, MA 02138 USA 


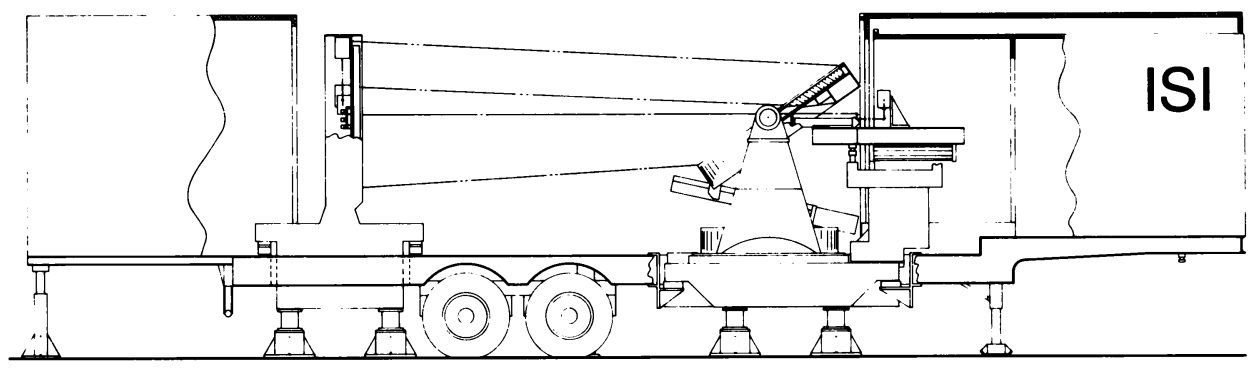

ÚCB INFRARED SPATIAL INTERFEROMETER 1 METER

SEMI-TRAILER OUTLINE

Fig. 1. Schematic of one of the ISI 1.65 meter telescopes mounted in a trailer.

the integrated signal to noise ratio would have been improved by approximately a factor of 56. Even with poor seeing, heterodyne detection and measurement of the fringe power over the entire bandwidth can allow an accurate evaluation of visibility, which is proportional to the square root of the ratio of the power in the entire fringe spectrum divided by the product of powers in the two signals detected by the heterodyne detectors of each telescope. Even though the incoming wave may not be all in phase at the telescope aperture, only that part which is in phase mixes properly with the rather pure wave front of the laser local oscillator and is detected in the heterodyne detector. It is this same in-phase component which produces the fringe, so that the ratio of the two gives an accurate measurement of visibility regardless of seeing.

We have extensive measurements of the fluctuations found in the relative pathlengths from a star to the two telescopes, and in the 10 meter pathlength within each telescope which is monitored by laser interferometers. These show substantial deviations from the simple Kolmogorov-Taylor model which has generally been used as a first approximation for atmospheric fluctuations. Some of these results have been previously reported by Bester et al. (1992), and others are discussed briefly in the paper by Bester et al. in this volume. The results show outer scales for the turbulence which are as low as about 10 meters under conditions of good seeing. Fortunately, they also indicate that deviations from the standard model make more favorable than previously expected the use of long baselines, of infrared wavelengths, and of adaptive optics.

\section{Stellar observations}

We have earlier reported some high angular resolution results on IRC $+10216, \alpha$ Orionis, and $o$ Ceti (Danchi et al., 1990; Bester et al., 1991). Visibility measurements on 13 stars including these three demonstrate that: (1) dust is formed within a few stellar radii of the star and at temperatures as high as $1300 \mathrm{~K}$; $(2)$ in the case of long period variables, new dust is characteristically formed during the cooling cycle close to the star; (3) while generally long period variables roughly repeat visibility 


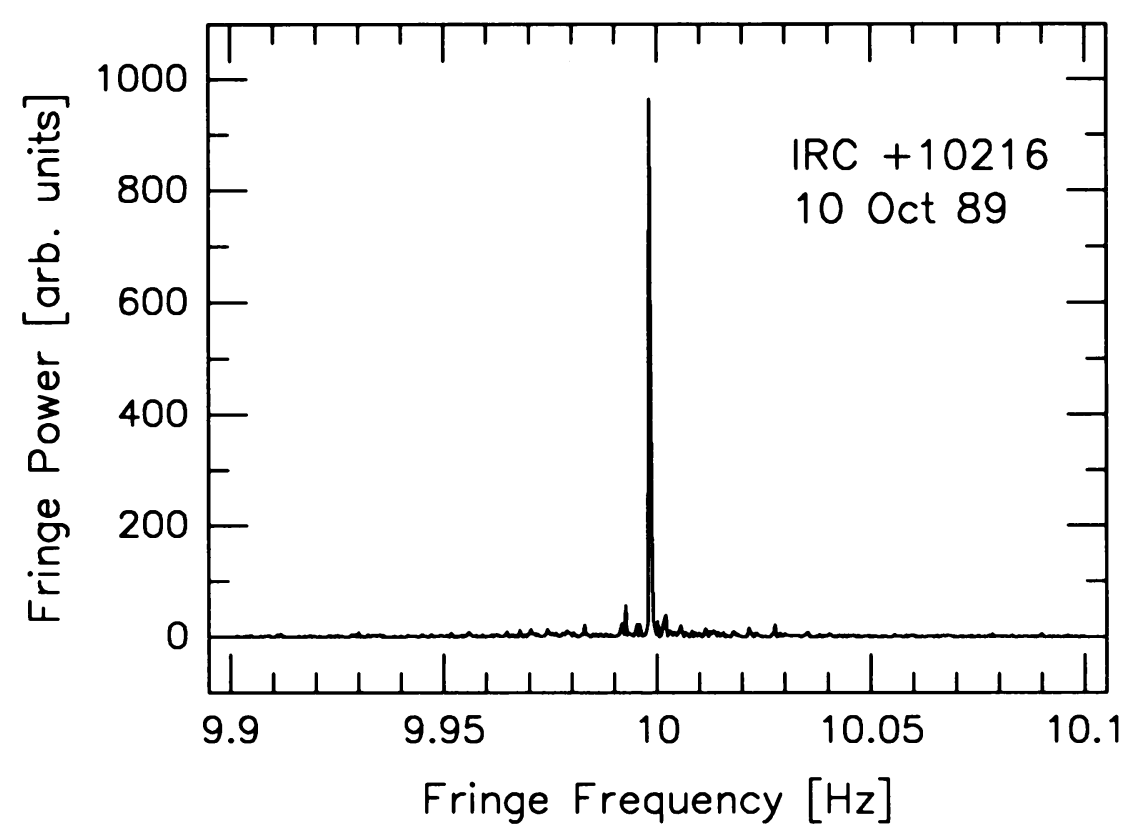

Fig. 2. Fringe signal resulting from 2621 seconds of observation of IRC +10216 under very good seeing conditions on October 10, 1989. The central spike is as narrow in frequency as the resolution determined by the length of observation, $3.8 \times 10^{-4} \mathrm{~Hz}$. Weak sidebands due to atmospheric fluctuations spread out over about $0.04 \mathrm{~Hz}$.

characteristics as a function of phase of their luminosity, at least in some cases the visibility is substantially different at the same phase during different cycles; (4) a substantial fraction of stars ( 6 of the 13 observed) have dust shells which are a considerable distance from the star and indicate that dust is formed episodically rather than continuously or on each cycle of luminosity.

The conclusion that some stars emit gas and dust episodically is well illustrated by the case of $\alpha$ Orionis, for which the visibility curve is shown in Figure 4 . The approximate flattening out of the visibility curve for spatial frequencies larger than about $1.0 \times 10^{5}$ cycles $\mathrm{rad}^{-1}$ indicates that essentially all of the dust has been resolved at that resolution and that there is no appreciable dust between about one arc sec from the star and the stellar disk itself. The strong minimum at a spatial frequency of about $1.2 \times 10^{5}$ and the maximum at about $2.4 \times 10^{5}$ cycles $\operatorname{rad}^{-1}$ represent an interference between fringes from the central star and those from the inner warm surface of the dust shell, and thus provide a good measure of the shell's position, at a radius of about $1.0 \mathrm{arc} \mathrm{sec}$ from the star. The height of this maximum, and of further oscillations in the visibility curve at higher resolutions, indicate that the dust shell is rather thin, perhaps only about 0.1 or 0.2 arc sec thick. Spectroscopic measurements (Bernat et al., 1979) in the near IR indicate that 


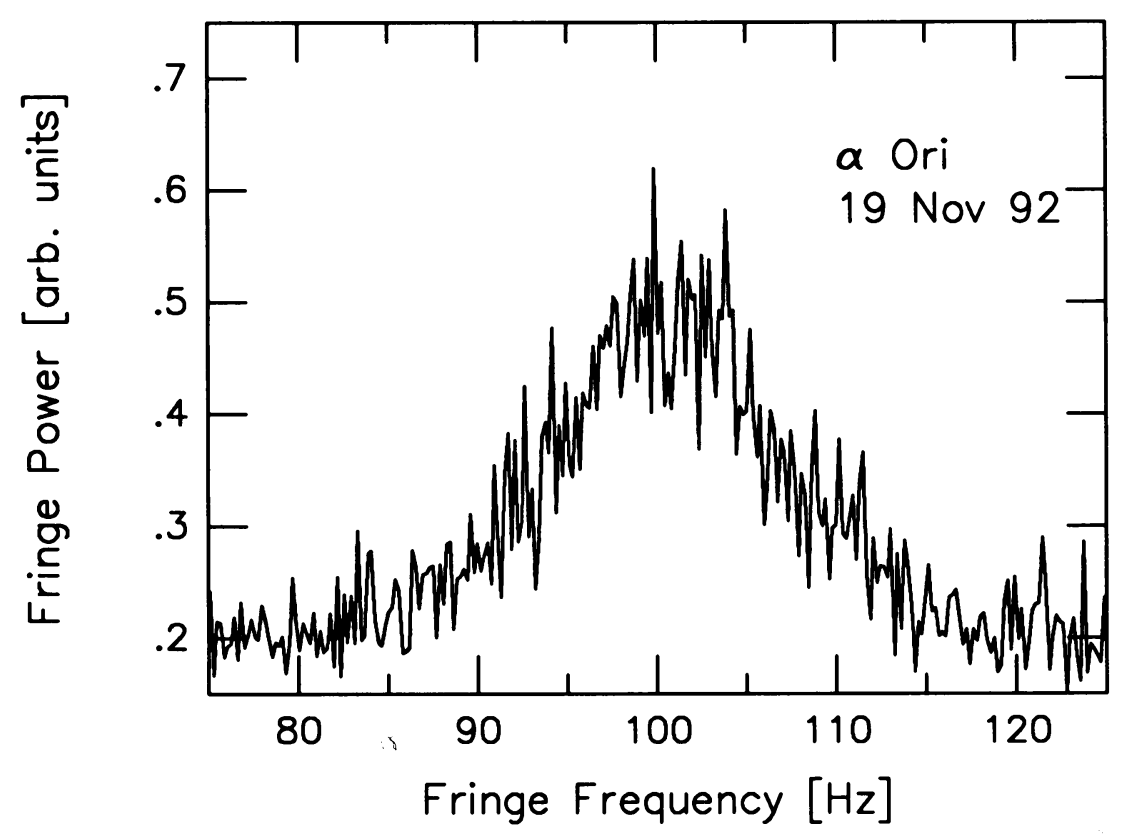

Fig. 3. Fringe signal resulting from 262 seconds of observation of $\alpha$ Ori under very poor seeing conditions on November 19, 1992. The fringe signal is spread out over as much as $30 \mathrm{~Hz}$ due to atmospheric pathlength fluctuations.

there are two batches of gas surrounding $\alpha$ Orionis, with velocities $11 \mathrm{~km} \mathrm{~s}^{-1}$ and 18 $\mathrm{km} \mathrm{s}^{-1}$ and temperatures of about $270 \mathrm{~K}$ and $70 \mathrm{~K}$ respectively. The velocity of the warmer shell, the position of which is measured here, and the history of fluctuations in the luminosity of $\alpha$ Orionis give a strong indication that the shell was emitted during the exceptional fluctuations of the mid 1940's, and hence predict a distance to $\alpha$ Orionis of about $110 \mathrm{pc}$. The common occurrence of such episodic emission of dust interspersed with long periods of very little emission is indicated by the six stars which have dust shells quite removed from the star itself as given in the table of stellar results of Danchi et al. in this volume. These results also allow a rather reliable calculation of the rate of mass loss from each of the stars.

Visibility curves for $o$ Ceti are shown in Figure 5 for the star at maximum luminosity on two different occasions. As is evident from the figure, the visibility curves are different for these two different cycles, one in 1989 and the other in 1992. o Ceti also happens to be a case where the visibility curve indicates the presence of dust quite close to the star and formed at a temperature near $1300 \mathrm{~K}$.

\section{Future plans}

While an active program of observation of atmospheric fluctuations, stellar visibilities, and astrometry continues with the ISI, we look forward to three major possible 


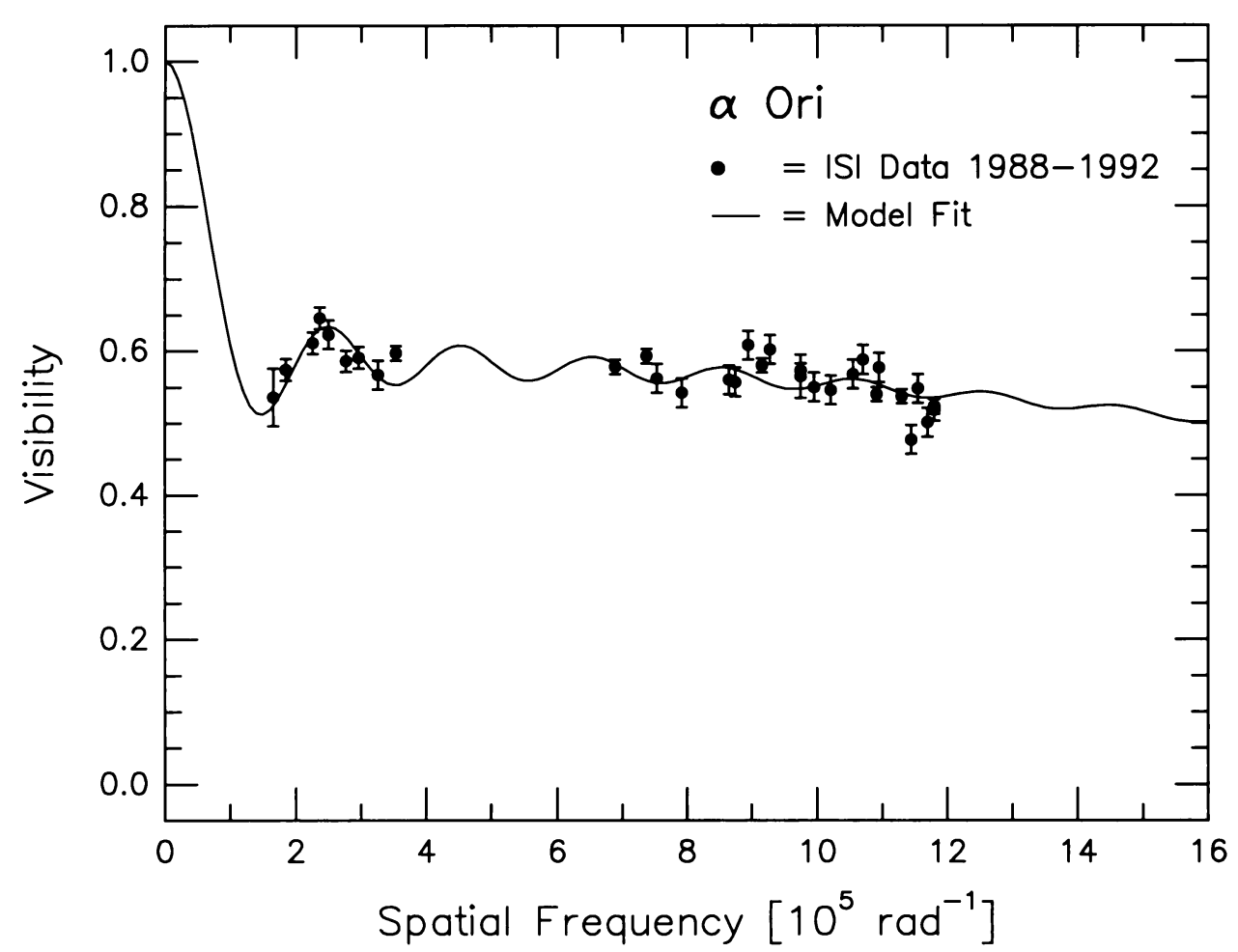

Fig. 4. The observed visibility curve of $\alpha$ Ori as a function of spatial frequency of the interference pattern on the sky. The relative constancy of visibility for spatial frequencies $>1.0 \times 10^{5}$ cycles $\mathrm{rad}^{-1}$ indicates there is very little dust at distances closer than about $1 \mathrm{arc}$ sec. The positions of the first minimum and maximum in the sinusoidal variation which decreases in amplitude towards higher spatial resolution indicates that the inner surface of the dust shell is at 1.0 arc sec radius from the star.

improvements in the next few years. The first and easiest one is for RF filters to be inserted which will narrow the bandwidth and allow measurements of visibilities on molecular absorption lines produced within the dust shells of stars. This should allow location of where particular molecules are formed as well as their abundance, distribution, and states of excitation and thus contribute to the understanding of basic questions about molecular formation. A second improvement, but one which is major and much more expensive, would be to obtain a third telescope of the same type which will allow phase closure and much faster observations at a wide variety of baselines. We would then also increase the baseline lengths to a maximum of 65 meters, yielding angular resolutions as fine as 0.017 arc sec. Another addition to this system which is very desirable is to use infrared delay lines which will allow direct detection. While both heterodyne and direct detection have advantages and 


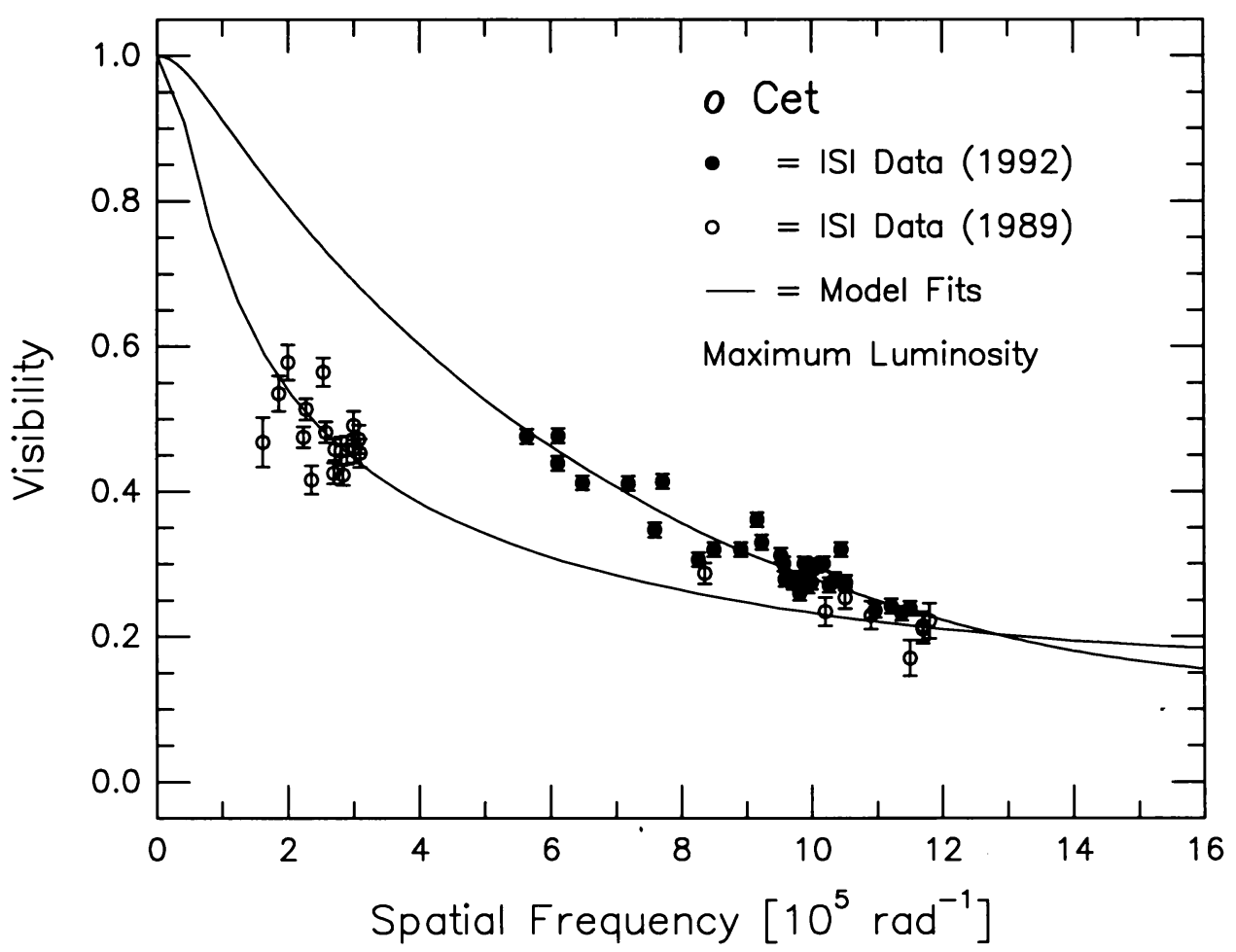

Fig. 5. The observed visibility curves of $o$ Ceti during maximum luminosity in 1989 and in 1992. These show substantial differences in visibility and hence in the distribution of dust at the same phase of the two different cycles of variation.

can complement each other, the special advantage of direct detection is that it will circumvent the quantum noise inherent in heterodyne detection and also allow a considerable increase in bandwidth. This should improve the sensitivity by about 5 mag. The ISI's present sensitivity will allow detection of infrared stars 5 mag weaker than the most intense ones such as $\alpha$ Orionis, but the additional sensitivity which can be provided by direct detection will make worthwhile the addition of infrared delay lines for use on still weaker stars.

\section{Acknowledgements}

This work was mainly supported by the United States Office of the Chief of Naval Research grant N00014-89-J-1583 and a grant from the National Science Foundation. This research has made use of the Simbad database, operated at CDS, Strasbourg, France. 


\section{References}

Bernat, A.P., Hall, D.N.B., Hinkle, K.H., and Ridgway, S.T.: 1979, Astrophys. J. (Lett.) 233, L135

Bester, M., Danchi, W.C., Degiacomi, C.G., Greenhill, L.J., and Townes, C.H.: 1992, Astrophys. J. 392,357

Bester, M., Danchi, W.C., Degiacomi, C.G., Townes, C.H., and Geballe, T.R.: 1991, Astrophys. J. (Lett.) 367, L27

Bester, M., Degiacomi, C.G., Danchi, W.C., Greenhill, L.J., Townes, C.H., Reisinger, A., and Weaver, J.: this volume

Danchi, W.C., Bester, M., Degiacomi, C.G., McCullough, P.R., and Townes, C.H.: 1990, Astrophys. J. (Lett.) 359, L59

Danchi, W.C., Greenhill, L.J., Bester, M., Degiacomi, C.G., and Townes, C.H.: this volume

\section{Discussion:}

White:

What is the height above ground of your laser metrology, and what other local effects might cause the 'seeing' fluctuations?

Townes:

The height is about 10 feet above ground. On Mt. Wilson there are, of course, trees and buildings which can affect turbulence, but I believe nothing else of note. 